\title{
Rapid Review and Synthesis of the Palliative Care Matters Scientific Evidence
}

\author{
Michelle Sims, $\mathrm{PhD}^{1}$ and Konrad Fassbender, MA, $\mathrm{PhD}^{1,2}$
}

\begin{abstract}
Background: This article details the methods used for a rapid literature review.

Objective: The eight scientific articles contained in this Palliative Care Matters supplemental issue synthesize and interpret evidence from the rapid review process outlined in this study.

Methods: The methods of the rapid review were adopted from the Preferred Reporting Items for Systematic Reviews and Meta-Analyses (PRISMA-P) statement of PRISMA.

Results: The current article contains the literature search strategies for both gray and academic literature, resource eligibility criteria for inclusion and exclusion, and tables and figures to outline the literature search process, and delimit the number of resource items captured at each step in the process.
\end{abstract}

Keywords: consensus development conference; literature review methods; palliative care; PRISMA; rapid review

\section{Introduction}

\section{Rationale and objectives}

T his article Details the methods used in the rapid review of the academic and gray literature for all eight scientific evidence articles in this Palliative Care Matters supplement. The Palliative Care Matters Research Support Team, headed by the lead scientific researcher (second author on this article), opted for the rapid review methodology of literature search due to the magnitude of the Palliative Care Matters project. Due to their quick turnaround, the rapid review method is often preferred in policy and decision-making research, particularly in healthcare. ${ }^{1}$ Since the Palliative Care Matters project was launched to affect healthcare policy change, it was well suited for the rapid review methodology.

The goal of the Palliative Care Matters rapid review process was to capture as much of the literature on each of the eight scientific topics as possible in a short time frame (the review for all eight topics was completed three months). Within the methodological continuum of assessing evidence, the rapid review method poses a trade-off between time cost and literature-searching scope. To evaluate the evidence in a timely manner, it is necessary to limit the amount of literature searching and assessment.
The Palliative Care Matters Research Support Team conducted the review and consisted of two information scientists, one research coordinator, three research analysts, and the lead scientific researcher (see Acknowledgments). Each team member's contribution to the rapid review process is outlined in the Methods section.

\section{Methods}

This rapid review was performed according to the Preferred Reporting Items for Systematic Reviews and MetaAnalyses (PRISMA-P) 2015 guidelines of PRISMA. ${ }^{2}$

\section{Literature search and screening process, information sources, and search strategies}

An overview of the four-step literature search and screening process is illustrated in Figure 1. The supplementary data contains PRISMA diagrams for each of the eight Palliative Care Matters scientific articles contained in this supplemental issue. The diagrams give the flow and number of resources captured at steps 1-4:

Step 1: Initial literature searches. A team of information scientists performed an initial literature search. Searches

\footnotetext{
${ }^{1}$ Covenant Health Palliative Institute, Gray Nuns Community Hospital, St. Marguerite Health Services Center, Edmonton, Alberta, Canada. ${ }^{2}$ Department of Oncology, University of Alberta, Edmonton, Alberta, Canada.

Accepted August 22, 2017.
} 


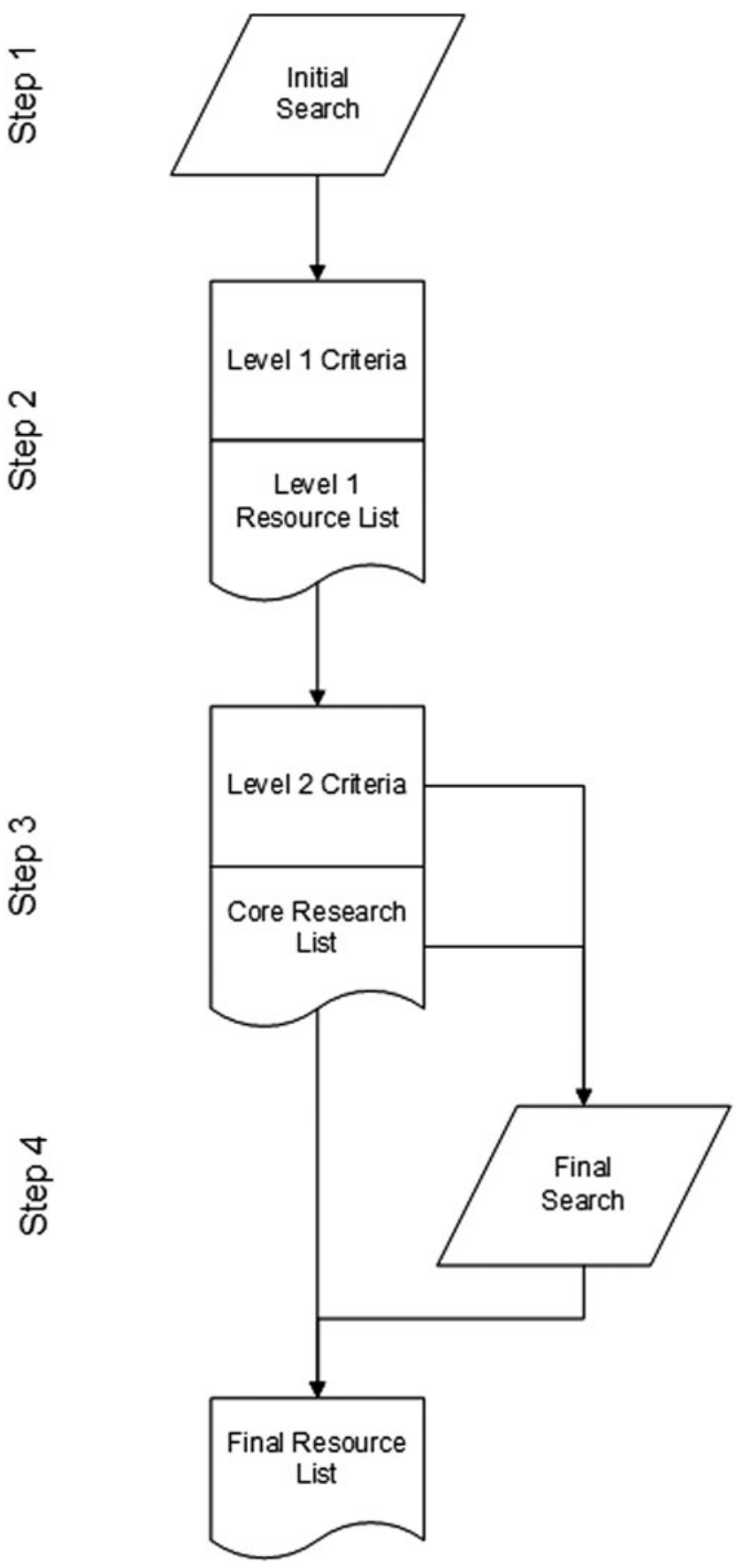

FIG. 1. Preferred Reporting Items for Systematic Reviews and Meta-Analyses diagram overview of four-step literature search and screen process.

for each scientific article were conducted independently from one another. Separate search sources and search strategies were developed for two domains of literature: published articles, and gray literature (including theses, policy documents, and reports). Initial search strategies were developed jointly by the information scientists and the lead scientific researcher (second author on this article). Search strategies were based on the rationale and objectives for each article outlined in the Introduction section. Search sources for published articles included MEDLINE, CINAHL, and Embase. Search sources for the gray literature include Proquest
Dissertations and Theses Global, CareSearch Gray Literature, Cochrane Palliative Care Library, Google Scholar, and Google. Additional sources for the initial literature search were provided by the lead scientific researcher. Citations and abstracts were downloaded into Endnote ${ }^{3}$ libraries and exported to text documents for the following step. This initial literature search yielded 6500 resources across all scientific articles.

Step 2: Level 1 criteria and resource lists. A team of research analysts (first author on this article is one analyst) set the first level of inclusion and exclusion criteria to inform their first screen of the initial literature search. The lead scientific researcher abstained from this level of literature screening, except to resolve criteria queries. Any uncertainties the research analysts had about the inclusion or exclusion of a resource were brought to the lead scientific researcher for decision. If warranted, the inclusion/exclusion criteria were adjusted accordingly. This Level 1 literature screen yielded 812 resources across all scientific articles.

Step 3: Level 2 criteria and core resource lists. For each scientific article, the primary criteria and resulting literature screen were then reviewed by the lead scientific researcher and the article's scientific expert, resulting in a secondary, more refined, set of criteria and literature screen. Any inconsistencies and disagreements in criteria and literature selection were resolved by consensus, with the article's scientific expert having the final decision.

Scientific experts were also given the opportunity to provide additional resources to add to the secondary literature screen. These resources included additional published articles, websites, reports, and policy documents. With the expert-added resources, the Level 2 literature screens serve as each article's core resources list. The core lists combined across articles contained 523 resources.

Step 4: Final literature searches. The information scientists and research analysts performed a final literature search for each scientific article based on the core resources lists. The final literature search served to gather more recent and in-progress literature/research to supplement the core list. This was achieved through three additional searches: for each resource on the core list,

1. the first author was contacted if author and contact information for the author was readily available;

2. stakeholders (spanning nonprofit, government, and for-profit sectors) were identified and contacted; and

3. citing literature was identified through a Scopus search.

These additional resources were added to the Endnote ${ }^{3}$ libraries to remove duplications with the initial literature search. After removing duplications, the final literature search yielded 5545 additional resources across all scientific articles.

For each scientific article, those additional resources that fit within the Level 2 criteria (as assessed by the research analysts) were combined with the core list to produce a final resource list. The final resources lists combined across articles contained 787 resources.

Full-text articles for each resource in the final bibliography were obtained and forwarded to the scientific experts to aid in their review of the scientific evidence. Each of the scientific 
Table 1. Final (Level 2) Inclusion and Exclusion Criteria for Each Topic

Inclusion criteria Exclusion criteria

Scientific Article No. 1: Integrated palliative care

1. Palliative Care

2. Hospice Care

3. Hospice(s)

4. Attitudes to Death

5. Continuity of patient care/organization and administration

6. Community health services/organization and administration

7. Community networks/organization and administration

8. Child health services/organization and administration

9. Life support care

10. Death

11. Delivery of healthcare

12. End-of-life care

13. Integrated/organization and administration

14. Integrat*

15. Coordinat*

16. Healthcare quality

17. Health services for the aged/organization and administration

18. Healthcare reform

19. Health policy

20. Interdisciplinary/multidisciplinary team and communication

21. State medicine/organization and administration

22. Models, organizational

23. Mental health services/organization and administration

24. National health programs/organization and administration

25. Patient-centered care

26. Person-centered care

27. Access

28. Patient perspective

29. Primary healthcare/organization and administration

30. Patient care team/organization and administration

31. Terminal care: Terminally ill

32. Resuscitation

33. Advance care planning

34. Bereavement

Scientific Article No. 2: Palliative care campaigns and awareness strategies

1. Effectiveness evaluation

2. Definition of what constitutes an "awareness campaign"

3. Dying well

4. Advance care planning

5. Health message

6. Literacy

7. Assessment and description of campaign elements' effectiveness

8. Inventory of approaches (international review)

9. Inventory of mediums; for example, print, visual, social media, etc. (survey)

10. Assessment of outcome measures

11. Recommendations to assess quality and success

Scientific Article No. 3: Palliative homecare

1. In-home

2. Advanced illness

3. Palliative care

4. End of life

5. Hospice care

6. Reviews

7. Empirical: experimental or observational

8. Clinical trials and other types of quantitative research

9. Terminal care

10. Assessment

Scientific Article No. 4: Palliative care education for caregivers
1. Palliative
2. End of life
3. Education

1. Homecare

2. Model specific (not incl. USA)

3. Health outcome indicator specific

4. Complementary/alternative medicine

5. Pathways/advance care planning/goals of care

6. Practice guidelines

7. Education

8. Discipline limiting (e.g., Cardiology, lung, etc.)

1. Doctors' discussions with patients

2. Healthcare practitioners' attitudes

3. Bereavement

4. Screening/diagnosis

5. Disease treatment

6. Social work (and not health focused)

7. Healthcare competence evaluation

8. Clinical practices

9. Case study/report

10. Political debate

1. Countries other than Canada

2. Care not focused on the patient
1. Advance care planning/end-of-life discussions

2. Editorials 
Table 1. (Continued)

\begin{tabular}{|c|c|}
\hline Inclusion criteria & Exclusion criteria \\
\hline $\begin{array}{l}\text { 4. Training } \\
\text { 5. Mentoring } \\
\text { 6. Resources } \\
\text { 7. Caregiver } \\
\text { 8. North America, Europe, The United Kingdom, Australia } \\
\text { 9. Evaluations of projects or pilot programs } \\
\text { 10. Organizational or governmental reports } \\
\text { 11. Specific conditions that pertain to end-of-life care or chrol }\end{array}$ & $\begin{array}{l}\text { 3. Opinion pieces } \\
\text { 4. Tool evaluations and measurements } \\
\text { 5. Knowledge of or comfort with end-of-life } \\
\text { care } \\
\text { 6. Standards of practice }\end{array}$ \\
\hline $\begin{array}{l}\text { cientific Article No. 5: Palliative care education for nurses and } \\
\text { 1. Palliative } \\
\text { 2. End of life } \\
\text { 3. Education } \\
\text { 4. Training } \\
\text { 5. Mentoring } \\
\text { 6. Resources } \\
\text { 7. Nurses (RN, LPN, unregulated, NP) } \\
\text { 8. Competency frameworks } \\
\text { 9. North America, Europe, The United Kingdom, Australia } \\
\text { 10. Evaluations of projects or pilot programs } \\
\text { 11. Organizational or government reports } \\
\text { 12. Chronic illness }\end{array}$ & $\begin{array}{l}\text { istants } \\
\text { 1. Advance care planning/end-of-life } \\
\text { discussions } \\
\text { 2. Editorials } \\
\text { 3. Opinion pieces } \\
\text { 4. Tool evaluations and measurements } \\
\text { 5. Knowledge of or comfort with end-of-life } \\
\text { care } \\
\text { 6. Standards of practice } \\
\text { 7. Focused strictly on specialty areas } \\
\text { (oncology, critical care, pediatrics, } \\
\text { geriatrics, heart failure) }\end{array}$ \\
\hline $\begin{array}{l}\text { Scientific Article No. 6: Palliative care education for physicians } \\
\text { 1. Palliative } \\
\text { 2. End of life } \\
\text { 3. Education } \\
\text { 4. Training } \\
\text { 5. Mentoring } \\
\text { 6. Resources } \\
\text { 7. Physician } \\
\text { 8. End-of-life discussions } \\
\text { 9. Competency frameworks } \\
\text { 10. North America, Europe, The United Kingdom, Australia } \\
\text { 11. Evaluations of projects or pilot programs } \\
\text { 12. Organizational or government reports }\end{array}$ & $\begin{array}{l}\text { 1. Editorials } \\
\text { 2. Opinion pieces } \\
\text { 3. Tool evaluations and measurements } \\
\text { 4. Knowledge of or comfort with end-of-life } \\
\text { care } \\
\text { 5. Standards of practice } \\
\text { 6. Focused strictly on specialty areas } \\
\text { (oncology, critical care, pediatrics, } \\
\text { geriatrics, heart failure) }\end{array}$ \\
\hline $\begin{array}{l}\text { Scientific Article No. 7: Palliative care frameworks } \\
\text { 1. Frameworks } \\
\text { 2. Policy } \\
\text { 3. Regulations } \\
\text { 4. Goal setting } \\
\text { 5. Action planning } \\
\text { 6. Evidence-based } \\
\text { 7. Cross-sectional survey } \\
\text { 8. Questionnaire } \\
\text { 9. Criteria/definitions for success }\end{array}$ & $<$ none $>$ \\
\hline $\begin{array}{l}\text { Scientific Article No. 8: Palliative care indicators } \\
\text { 1. Surveillance } \\
\text { 2. Barriers } \\
\text { 3. Facilitators } \\
\text { 4. Measurement challenges } \\
\text { 5. Population/patient-based } \\
\text { 6. Quality measurement } \\
\text { 7. Psychometrics } \\
\text { 8. Tool kits and models } \\
\text { 9. Indicator testing protocol } \\
\text { 10. Audit tools } \\
\text { 11. Systems approach } \\
\text { 12. Satisfaction } \\
\text { 13. Outcome } \\
\text { 14. Measures } \\
\text { 15. Feedback } \\
\text { 16. Benchmark } \\
\text { 17. Advanced care planning }\end{array}$ & $\begin{array}{l}\text { 1. Homecare } \\
\text { 2. Utilization (cost) } \\
\text { 3. Noncommunicable diseases } \\
\text { 4. Data sets (creation and comparison) } \\
\text { 5. Clinical practices } \\
\text { 6. Population scope limiting (e.g., pediatrics } \\
\text { only) }\end{array}$ \\
\hline
\end{tabular}

LPN, licensed practical nurse; NP, nurse practioner; RN, registered nurse. 
evidence articles in this supplement issue contains a discussion of the final bibliographic data items used for the review.

\section{Eligibility criteria}

The information scientists limited their search to English publications spanning the academic and gray literature. This yielded resources from Canada, the United States, the United Kingdom, Australia, Japan, Italy, among other countries. With few exceptions for key pieces of seminal research and policies/reports, the literature search was limited to the most recent 10 years (i.e., from 2006 to 2016). Resources were not limited in terms of setting of care, patient population or demographics, clinical interventions, nor disease. A summary of the final (Level 2) inclusion and exclusion criteria for each scientific article is given in Table 1.

Further discussions on the final resource lists' prioritization and outcomes, as well as the meta-analyses, are given in each scientific article within this supplemental issue.

\section{Acknowledgments}

The authors acknowledge the contributions of information scientists Sharna Polard and Roger Salus; research coordinator Heather Stiles; research analysts Viki Muller and Lisa Weisgerber; and scientific experts Kevin Brazil, James
Downer, Deborah Dudgeon, Sean Marrison, Barbara Pesut, Hsein Seow, Jane Seymour, and Allison Williams.

\section{Author Disclosure Statement}

No competing financial interests exist.

\section{References}

1. Garritty C, Stevens A, Gartlehner G, et al.: Cochrane Rapid Reviews Methods Group to play a leading role in guiding the production of informed high-quality, timely research evidence syntheses. Syst Rev 2016;5:184.

2. Moher D, Shamseer L, Clarke M, et al.: Preferred reporting items for systematic review and meta-analysis protocols (PRISMA-P) 2015 statement. Syst Rev 2015;4:1.

3. Clarivate Analytics: Endnote. Philadelphia, PA: Clarivate Analytics, 2016.

Address correspondence to:

Michelle Sims, PhD

Covenant Health Palliative Institute 1100 Youville Drive Edmonton, Alberta T6L 5X8

Canada

E-mail: michelle.sims@covenanthealth.ca 